ER R ATA

\title{
ERRATUM: When science falls from grace
}

S Krimsky

Nat. Med. 11, $591(2005)$

The last sentence of the book review should be replaced with the following: "It is a challenging, sometimes even prickly, book that explores the future direction of science from its flawed past. Judson asks us to consider whether scientific norms are undergoing a tectonic shift and whether such a shift is desirable." 